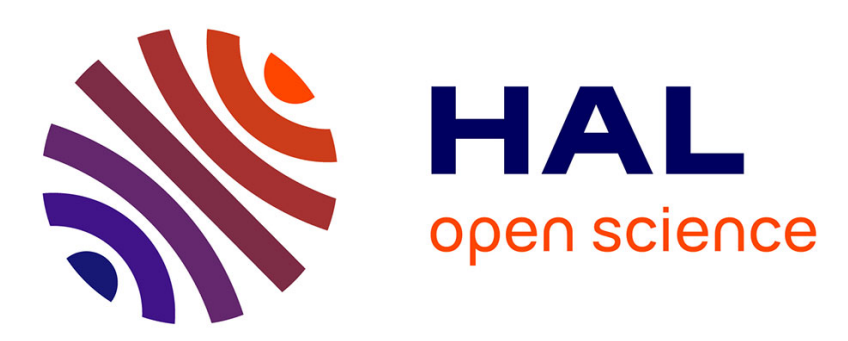

\title{
Melting curve and phase diagram of ammonia monohydrate at high pressure and temperature
}

H. Zhang, Frédéric Datchi, L. Andriambariarijaona, G. Zhang, J. A Queyroux, K. Béneut, M. Mezouar, S. Ninet

\section{- To cite this version:}

H. Zhang, Frédéric Datchi, L. Andriambariarijaona, G. Zhang, J. A Queyroux, et al.. Melting curve and phase diagram of ammonia monohydrate at high pressure and temperature. Journal of Chemical Physics, 2020, 153 (15), pp.154503. 10.1063/5.0021207 . hal-02990784

\section{HAL Id: hal-02990784 \\ https://hal.science/hal-02990784}

Submitted on 28 Sep 2021

HAL is a multi-disciplinary open access archive for the deposit and dissemination of scientific research documents, whether they are published or not. The documents may come from teaching and research institutions in France or abroad, or from public or private research centers.
L'archive ouverte pluridisciplinaire HAL, est destinée au dépôt et à la diffusion de documents scientifiques de niveau recherche, publiés ou non, émanant des établissements d'enseignement et de recherche français ou étrangers, des laboratoires publics ou privés. 


\title{
Melting curve and phase diagram of ammonia monohydrate at high pressure and temperature
}

\author{
H. Zhang, ${ }^{1, a)}$ F. Datchi, ${ }^{1}$ L. Andriambariarijaona, ${ }^{1}$ G. Zhang, ${ }^{1,2}$ J.A. Queyroux,${ }^{1}$ K. Béneut, ${ }^{1}$ M. Mezouar, ${ }^{3}$ and \\ S. Ninet ${ }^{1, b)}$ \\ 1) Institut de Minéralogie, de Physique des Matériaux et de Cosmochimie (IMPMC), Sorbonne Université, CNRS UMR 7590 , \\ IRD UMR 206, MNHN, 4, place Jussieu, Paris, France \\ ${ }^{2)}$ State Key Laboratory of Superhard Materials, Jilin University, Changchun 130012, China \\ 3) European Synchrotron Radiation Facility, BP 2220, F-38043 Grenoble Cedex, France
}

(Dated: 28 September 2021)

The phase diagram and melting behavior of the equimolar water-ammonia mixture have been investigated by Raman spectroscopy, x-ray diffraction and visual observations from $295 \mathrm{~K}$ to $675 \mathrm{~K}$ and up to $9 \mathrm{GPa}$. Our results show noncongruent melting behavior of AMH solid below $324 \mathrm{~K}$ and congruent melting at higher temperatures. The congruent melting is associated with the stability of a previously unobserved solid phase of AMH, which we named AMH-VII. Another, presumably water-rich, hydrate has also been detected in the range 4-7 GPa at $295 \mathrm{~K}$ on decompression of the high pressure DIMA phase. Comparing our melting data to literature suggests that non-congruent melting extends from $220 \mathrm{~K}$ to $324 \mathrm{~K}$, and that the solid phase which borders the fluid between 220 and $270 \mathrm{~K}$, called AMH-III, is not a proper phase of AMH but a solid solution of AHH and ice. These results allow us to propose a revised and extended experimental phase diagram of AMH.

\section{INTRODUCTION}

Water, ammonia, and methane are considered major components of the interiors of the giant icy planets Neptune and Uranus and their satellites, the icy satellites of Saturn (Titan, Enceladus) and Jupiter (Ganymede), and of the "Ocean" exoplanets recently discovered. Present models of the interior of Neptune and Uranus assume the existence of a thick layer composed of these ices submitted to extreme conditions of pressure (20-700 GPa) and temperature $(2000-6000 \mathrm{~K})^{1-6}$. Knowing the physical and chemical properties of these compounds over a large range of pressure $(\mathrm{P})$ and temperature $(\mathrm{T})$ is therefore needed to build robust interior models that can be compared to observations such as the magnetic field, the gravitational moments and the atmospheric composition, collected from Earth or space missions. In particular, the determination of their melting curve is important to determine whether the material is solid or fluid at a given depth inside the planet, with significant consequences on macroscopic properties such as the magnetic field.

In the past few decades, numerous investigations have been done on the high P-T properties of the pure ice compounds $\left(\mathrm{H}_{2} \mathrm{O}, \mathrm{NH}_{3}\right)$. These studies have revealed a rich polymorphism and several "exotic" solid phases, such as superionic

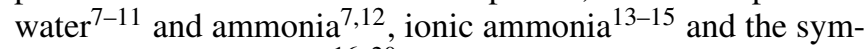
metric phase of water ${ }^{16-20}$. By contrast, the experimental study of water-ammonia mixtures has remained limited to modest pressures and low temperatures although these systems are a more realistic basis for planetary modelling than the single components.

At low temperature, water-ammonia mixtures crystallize in three different hydrates depending on the initial concentra-

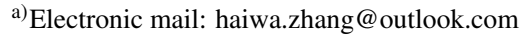

b)Electronic mail: sandra.ninet@ sorbonne-universite.fr
}

tion : ammonia monohydrate $\left(\mathrm{H}_{2} \mathrm{O}: \mathrm{NH}_{3}\right.$, noted $\left.\mathrm{AMH}\right)$, ammonia hemihydrate $\left(\mathrm{H}_{2} \mathrm{O}: 2 \mathrm{NH}_{3}\right.$, noted $\left.\mathrm{AHH}\right)$ and ammonia dihydrate $\left(2 \mathrm{H}_{2} \mathrm{O}: \mathrm{NH}_{3}\right.$, noted $\left.\mathrm{ADH}\right)$. The experimental phase diagram of $\mathrm{AMH}$ has been mostly investigated below $10 \mathrm{GPa}$ and for temperatures below ambient ${ }^{21,22}$. Six different solid phases have been reported in this $\mathrm{P}-\mathrm{T}$ range, noted AMH-I to $\mathrm{VI}^{21-25}$. At room temperature, two different solid phases have been observed: $\mathrm{AMH}-\mathrm{V}$, on one hand, obtained on compression of the fluid above $\sim 3.5 \mathrm{GPa}$, and AMH-VI, on the other hand, obtained upon warming above $270 \mathrm{~K}$ the low-temperature phase IV at $\sim 6.5 \mathrm{GPa}^{21}$. Wilson et al. ${ }^{25}$ have shown that AMH-V is, in fact, a solid mixture of the hemi-hydrate AHH-II and ice VII solids. AMH-VI has been initially described as a disordered molecular alloy (DMA) constructed by randomly positioning, with equal probability, water and ammonia molecules on a body-centered cubic lattice $^{21}$. More recently, it was shown that AMH-VI is rather composed of two phases $^{22}$ : first, a partially ionized $b c c$ phase baptized DIMA, similar to DMA but composed of about onethird ammonium and hydroxyl ions, and of two-third water and ammonia molecules; and second, a fully ionic and ordered $\mathrm{P} 4 / \mathrm{nmm}$ phase solely composed of $\mathrm{NH}_{4}^{+}$and $\mathrm{OH}^{-}$ions. The latter was previously predicted by theoretical calculations to be the ground state of $\mathrm{AMH}$ in this pressure range ${ }^{26}$, yet it has so far only been observed in minor quantity in experiments compared to the DIMA phase.

The phase diagram of AMH remains mostly unexplored by experiment above ambient temperature. Recent theoretical studies have predicted several new phases, and in particular the existence of plastic and superionic solids at high $\mathrm{P}-\mathrm{T}^{27,28}$. Experimental studies ${ }^{29,30}$ of the melting curve of AMH are also limited to temperatures below $400 \mathrm{~K}$ and lack consistency, calling for more experimental data.

In this work, we report the experimental measurements of the melting curve of AMH from $295 \mathrm{~K}$ to $675 \mathrm{~K}$ up to $9 \mathrm{GPa}$ by in situ Raman spectroscopy, $\mathrm{x}$-ray diffraction and visual observations. Our results show non-congruent melting be- 
havior of AMH solid below $324 \mathrm{~K}$ and congruent melting at higher temperatures. The congruent melting is associated with the stability of a previously unobserved solid phase of AMH, which we named AMH-VII. Our results also suggest that the solid phase which borders the fluid between 220 and $270 \mathrm{~K}$, called AMH-III, is not a proper phase of AMH but a solid solution of $\mathrm{AHH}$ and ice. A revised phase diagram of $\mathrm{AMH}$, extending to $675 \mathrm{~K}$ and $9 \mathrm{GPa}$, is thus proposed.

\section{EXPERIMENTAL METHODS}

Equimolar mixtures of water and ammonia were prepared in a stainless steel cylinder as described in Refs. [21, 22, and 25]. Ammonia gas (99.99\% purity) obtained from Air Liquide was condensed at liquid-nitrogen temperature into a preweighed stainless steel bottle, and the corresponding mass of deionized water was put into a second steel bottle to obtain a 1:1 molar mixture. Then, the two bottles were connected, and ammonia was allowed to condense in the water bottle immersed in liquid nitrogen. The final composition was checked by weighing the mixed solution and comparing with the sum of the masses of the two components. If the difference was larger than $1 \%$, the mixture was discarded and prepared again.

Samples of AMH were cryogenically loaded in membrane diamond anvil cells (MDAC), as described in Refs. [22 and 25]. The gaskets were made of rhenium. The sealed sample of AMH was cooled to $210 \mathrm{~K}$ using a mixture of isopropanol and liquid $\mathrm{N}_{2}$, in order to bring the mixture in the stable liquid domain at 1 bar, and thus prevent ammonia from evaporating. A drop of liquid AMH was then rapidly poured onto the rhenium gasket hole while the MDAC was held at $\sim 120 \mathrm{~K}$ above cold $\mathrm{N}_{2}$ gas. The MDAC was then quickly assembled and compressed to a pressure usually between 10 and $20 \mathrm{GPa}$ before letting the MDACs warm to room temperature. As reported in previous studies, this low-temperature (LT) compression leads to the formation of AMH-VI at room temperature ${ }^{21,22}$.

A commercial cylindrical resistive heater (Watlow) surrounding the body of the cell was used for heating up to $700 \mathrm{~K}$. The temperature of the sample was determined with a type-K thermocouple placed close to the diamond anvils. The temperature uncertainties are below $5 \mathrm{~K}$. A ruby ball was used to measure the pressure ${ }^{31}$. Pressure error bars were estimated from the standard deviation of the fitted luminescence peaks positions, and were at worst $0.37 \mathrm{GPa}$ at $675 \mathrm{~K}$ and 8.34 $\mathrm{GPa}$. We used ruby instead of the $\mathrm{SrB}_{4} \mathrm{O}_{7}: \mathrm{Sm}^{2+}$ sensor ${ }^{32}$ despite the lower accuracy of ruby at high temperatures because $\mathrm{SrB}_{4} \mathrm{O}_{7}: \mathrm{Sm}^{2+}$ is chemically attacked by liquid water above $550 \mathrm{~K}$, resulting in its total dissolution ${ }^{33}$. No evidence of chemical reaction has been observed here between the sample and the ruby ball or the rhenium gasket.

The melting line of AMH was determined by visual observations of the solid-fluid equilibrium. At each investigated $P$ $T$ point, we registered whether the sample was solid, liquid or at solid-liquid coexistence. For $T<460 \mathrm{~K}$, the melting points were obtained during isothermal compression or decompression runs. The melting pressure was defined as the average of the two pressures bracketing melting and the uncertainty as half the pressure interval. For $T>460 \mathrm{~K}$, the solid-fluid coexistence was maintained during the slow heating of the sample by simultaneously increasing the load. Pressure was measured when the temperature was stabilized and the uncertainty was taken as that of the ruby measurement. The latter method enables a precise measurement of the melting curve and prevents from the problems related to metastabilities such as undercooling or over-pressurization.

Raman experiments were carried out with an in-house confocal spectrometer. The excitation source is a continuous laser $\mathrm{Ar}^{+}$emitting at $514.5 \mathrm{~nm}$ focussed onto the sample with a Mitutoyo $20 \mathrm{X}$ objective to a spot of about $2 \mu \mathrm{m}$. The backscattered light was spatially filtered by a confocal pinhole, chromatically filtered by razor edge filters (Semrock), and then dispersed by an HR450 spectrograph (Horiba) coupled to a water-cooled CCD camera (Andor).

Angular dispersive $\mathrm{x}$-ray diffraction experiments were done at beamline ID27 of the European Radiation Synchrotron Facility (ESRF, Grenoble, France). The x-ray wavelength was $0.3738 \AA$. The focused $x$-ray beam has a spot size of $2.5 \times 3$ $\mu \mathrm{m}$ FWHM at ID27. Diffraction images were recorded on 2D detectors (MarResearch mar345 IP or marCCD). The detector distance and tilt, and the position of the $\mathrm{x}$-ray beam were calibrated using x-ray standards from NIST $\left(\mathrm{LaB}_{6}\right.$ or $\left.\mathrm{CeO}_{2}\right)$. The integration of the $\mathrm{x}$-ray images was performed with the fit $2 \mathrm{D}^{34}$ or Dioptas ${ }^{35}$ software programs. Le Bail or Rietveld refinements of the diffraction patterns were performed with the Fullprof software suite ${ }^{36}$.

\section{RESULTS}

The experiments reported here showed two different melting behaviour of the AMH solid, depending on whether temperature was below or above $324 \mathrm{~K}$. We start by presenting our observations made on decompressing solid AMH below $324 \mathrm{~K}$, which displays a non-congruent melting behaviour, and follow by presenting the congruent melting line measured above $324 \mathrm{~K}$. This change of behaviour is associated with the presence of a previously uncovered solid phase of $\mathrm{AMH}$ (called AMH-VII) above $324 \mathrm{~K}$.

\section{A. Observation and characterization of the non-congruent melting line $(T<324 \mathrm{~K})$}

Starting from a sample initially in phase AMH-VI at 8.35 $\mathrm{GPa}$, several solid-solid phases transitions were observed on decompression of the sample at 295 K. Figures 1(a) and (b) respectively show the evolution of the Raman spectrum and of the integrated $\mathrm{x}$-ray diffraction (XRD) pattern. As noted in the Introduction, AMH-VI is a mixture of the $b c c$ DIMA and $\mathrm{P} 4 / \mathrm{nmm}$ phases $^{22}$. The presence of the $\mathrm{P} 4 / \mathrm{nmm}$ phase is more easily observed in the Raman spectrum, with sharp lattice bands between 200 and $500 \mathrm{~cm}^{-1}$, and a strong $\mathrm{OH}$ stretching band at $3700 \mathrm{~cm}^{-1}$ from $\mathrm{OH}^{-}$ions, but less obvious in the XRD pattern which is dominated by the DIMA Bragg peaks. Below $7 \mathrm{GPa}$, large modifications of the Raman 

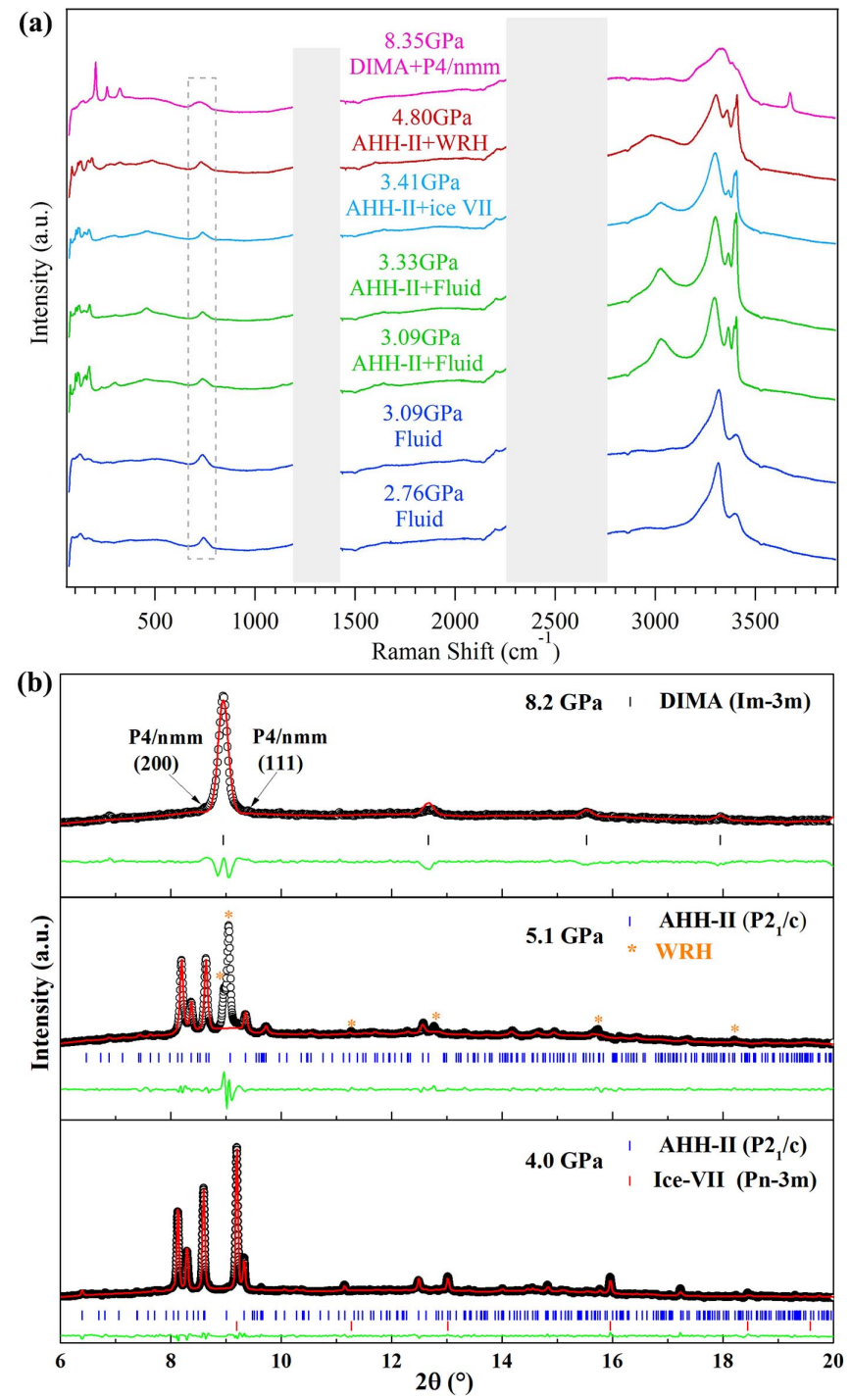

FIG. 1. (Color online)(a) Evolution of the Raman spectra of AMH during the decompression from $8.35 \mathrm{GPa}$ to $2.76 \mathrm{GPa}$ at room temperature. The frequency windows from $1200-1400 \mathrm{~cm}^{-1}$ to 2200 $2700 \mathrm{~cm}^{-1}$ are greyed as they are dominated by, respectively, the first- and second-order Raman signal from the diamond anvils. The peak around $750 \mathrm{~cm}^{-1}$ marked by the dotted frame comes from impurities in the diamond anvils. (b) X-ray diffraction patterns of AMH at room temperature collected upon decompression. The symbols are experimental data and the red lines are Le Bail refinement. Sticks show the positions of Bragg reflections. The green line represents the difference between observed and calculated profiles structure.

spectra and XRD pattern occur, as can be seen in the data collected at 4.8 and 5.1 GPa, respectively. First, the Raman peaks assigned to the $\mathrm{P} 4 / \mathrm{nmm}$ phase disappear; second, the shape of the Raman bands assigned to the $\mathrm{H}_{2} \mathrm{O}$ and $\mathrm{NH}_{3}$ molecule stretching between 2900 and $3500 \mathrm{~cm}^{-1}$ significantly change. These changes are consistent with those observed by infrared spectroscopy and are the result of a phase transition to a purely molecular phase $\mathrm{e}^{22}$. The XRD pattern collected at 5.1 GPa actually reveals the presence of two phases (see the Le
Bail fit in Figure 1(b) at $5.1 \mathrm{GPa}$ ): the molecular hemihydrate phase AHH-II, with $\mathrm{P} 2{ }_{1} / \mathrm{c}$ space group $[a=3.3235(14)$ $\AA, b=9.1708(59) \AA, c=8.8825(40) \AA$ and $\beta=94.38(5)$ as described in Ref.[25], and a second, unidentified hydrate. Since the latter coexists with $\mathrm{AHH}$, its composition must be richer in water than ammonia in order to comply with the initial equimolar composition of the sample; we thus named it waterrich hydrate (WRH).

As the pressure is further lowered, new changes occur both in the Raman spectrum and the XRD pattern. The XRD pattern collected at $4.0 \mathrm{GPa}$ is the same as that reported by Wilson et al. ${ }^{25}$ on freezing the $1: 1$ liquid at room temperature and identified as a mixture of AHH-II and ice VII. We could actually achieve a good Le Bail fit of this pattern using a mixture of AHH-II and ice VII, and the lattice parameters obtained for the $\mathrm{P} 2{ }_{1} / \mathrm{c}$ AHH-II $[a=3.3754(3) \AA, b=9.2292(9)$ $\AA, c=8.9687(7) \AA$ and $\beta=94.41(1)]$ and for Pn-3m ice VII $[a=$ $3.3088(1)] \AA)$ agree very well with Ref. [25]. Slight changes are also detected in the Raman spectrum in the $\mathrm{O}-\mathrm{H}$ and $\mathrm{N}-\mathrm{H}$ stretching modes (between 2900 and $3500 \mathrm{~cm}^{-1}$ ) at $3.41 \mathrm{GPa}$. However, it is difficult to distinguish the Raman spectra of the AHH-II + ice VII mixture from that of pure AHH-II, since the weak O-H stretch Raman modes of ice VII occur in the same frequency range as the strong $\mathrm{N}-\mathrm{H}$ stretch modes of $\mathrm{AHH}-\mathrm{II}$ (see Fig. 2 of the $\mathrm{SM}^{37}$ ). By contrast, this transition is easily detected by visual inspection of the sample as illustrated in Figure 2: the AHH-II + WRH mixture is transparent at 4.8 GPa [Fig.2(a)], whereas the sample adopts a granular aspect when WRH dehydrate into AHH-II + ice VII [Fig.2(b)] at 3.41 $\mathrm{GPa}$.

The sample images reported in Figure 2 show that the sample composed of AHH-II + ice VII starts to melt (coexistence of a solid and a fluid phase) at $3.33 \mathrm{GPa}$ (Fig.2(c)): the crystalline grains gather and start to move in their liquid while the pressure remains stable, as seen in Figures 2(d) and 2(e). The amount of fluid is too small at this pressure to be detectable by Raman, and the Raman spectra taken at different positions resemble that of the AHH-II+ice VII mixture [Fig.1(a)]. Upon further decreasing the load, the sample pressure also decreases and the amount of fluid phase increases [see Fig. 2(f) at 3.09 $\mathrm{GPa}$ and 2(g) at 2.84 GPa)]. The collected Raman spectra from the solid phase is consistent with AHH-II, and the one of the fluid phase is consistent with that reported in Ref. [29] at $2.77 \mathrm{GPa}, 295 \mathrm{~K}$. Finally, the sample becomes homogeneous and transparent again at $2.76 \mathrm{GPa}$ when it is fully fluid [Fig.2(h)], as confirmed by its Raman spectrum.

In conclusion, at $295 \mathrm{~K}$, the solid phase before melting is a mixture of AHH-II and ice VII and a non-congruent melting occurs: at the solidus pressure $(3.33 \mathrm{GPa})$, ice VII melts first and AHH-II coexists with the fluid down to the liquidus pressure $(2.76 \mathrm{GPa})$. The same observations were made along a decompression isotherm at $310 \mathrm{~K}$, where the solidus and liquidus pressure were measured at 3.04 and $3.55 \mathrm{GPa}$, respectively. 


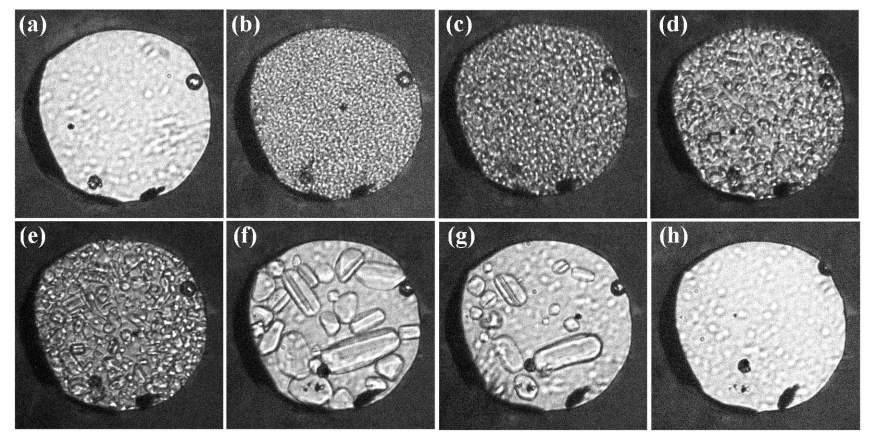

FIG. 2. Photographs of AMH sample during decompression from $4.80 \mathrm{GPa}$ to $2.76 \mathrm{GPa}$ at $295 \mathrm{~K}$. (a) $4.80 \mathrm{GPa}$ : solid mixture of AHHII+hydrate (the non homogeneous aspect come from dust particles on the camera) ; (b) $3.41 \mathrm{GPa}$ : solid mixture of AHH-II+ice-VII; (c), (d) and (e): $3.33 \mathrm{GPa}$, initial stage of sample melting;(f) and (g): crystals of AHH-II coexisting with the fluid at 3.09 and $2.84 \mathrm{GPa}$, respectively; (h) $2.76 \mathrm{GPa}$ : fully fluid sample.

\section{B. Congruent melting line $(T>324 \mathrm{~K})$ and evidence for a new high temperature phase (AMH-VII)}

From $324 \mathrm{~K}$ to $700 \mathrm{~K}$, a different melting behavior than described in the previous section was observed. This is illustrated in Figures 3A and 3B, which respectively show the photographs of the sample during decompression at $376 \mathrm{~K}$ and the measured Raman spectra. At $6.7 \mathrm{GPa}$, the sample was a mixture of AHH-II + WRH phases. The visual aspect of the sample is that of a polycrystal. As the pressure is decreased to $5.83 \mathrm{GPa}$, the sample image suddenly becomes more transparent (Fig.3A(b)). The Raman spectra confirms the occurence of a phase transition and reveals an homogeneous sample: whatever the probed sample region, we observed the disappearance of the mode around $2900 \mathrm{~cm}^{-1}$ associated to O-H stretching, the merging of the $\mathrm{N}-\mathrm{H}$ stretching peaks into a broad band at $3100-3500 \mathrm{~cm}^{-1}$, and the disappearance of the lattice modes. This Raman spectrum doesn't correspond to any reported solid phases of $\mathrm{AMH}$, thus revealing the existence of a new phase of $\mathrm{AMH}$ at high temperature, referred hereafter as AMH-VII. On further decompression, a progressive fragmentation of the AMH-VII solid occurs (see Fig.3A(c)) and a solid-fluid coexistence is detected at $4.45 \mathrm{GPa}$ [see images $3 \mathrm{~A}(\mathrm{~d})$ and $3 \mathrm{~A}(\mathrm{e})]$. The interface between the solid and fluid phase is however more difficult to observe than at room temperature, which indicates that the difference in refractive indices between the fluid and solid phase at high temperature is small. Contrary to the room temperature observations, the measured pressure does not change while varying the load at liquid-solid coexistence pressure (4.45 GPa at $376 \mathrm{~K}$ ), only the respective amount of fluid and solid phase varies. This demonstrates that (1) the melting is congruent at $376 \mathrm{~K}$, and (2) the solid phase has the same composition as the fluid, i.e., 1:1, and thus AMH-VII is a proper AMH solid phase. It can also be observed from Fig.3B that the Raman spectra of AMH-VII is very similar to that of the fluid phase, and it is thus difficult to infer melting solely from the Raman measure-
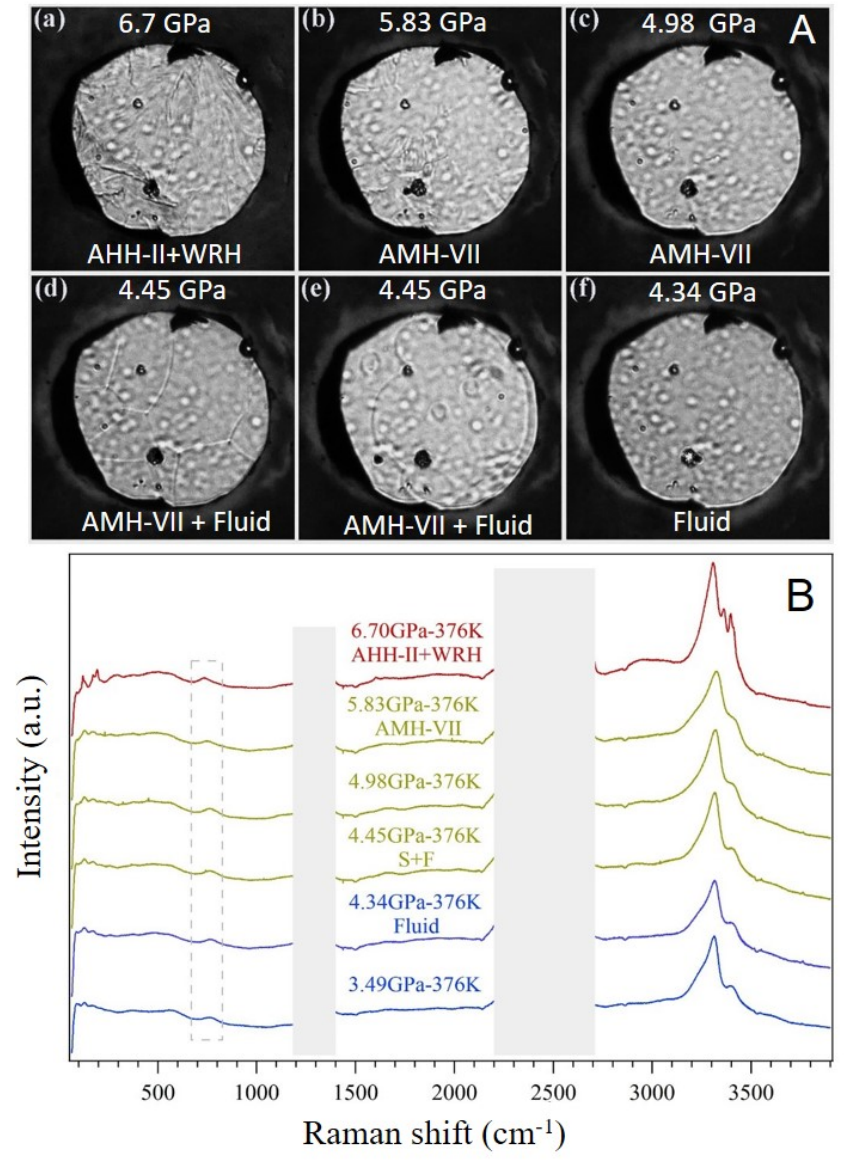

FIG. 3. (Color online) (A): Photographs of AMH from solid to fluid at $376 \mathrm{~K}$. The non homogeneous aspect (small white spots) of the background come from dust particles on the camera. (B): Evolution of the Raman spectra of AMH from solid (6.7 GPa) to fluid (3.49 $\mathrm{GPa})$ at $376 \mathrm{~K}$. The frequency windows $1200-1400 \mathrm{~cm}^{-1}$ to $2200-$ $2700 \mathrm{~cm}^{-1}$ are greyed as they are dominated by, respectively, the first- and second-order Raman signal from the diamond anvils. The peak around $750 \mathrm{~cm}^{-1}$ marked by the gray dotted frame comes from impurities in the diamond.

ments.

The equilibrium between AMH-VII and the fluid was observed from $324 \mathrm{~K}(3.59 \mathrm{GPa})$ up to $675 \mathrm{~K}(8.34 \mathrm{GPa})$. The $(\mathrm{P}, \mathrm{T})$ conditions which define the congruent melting line are reported in Figure 5 with red symbols and presented in the Table I. In this P-T range, the melting pressure is a monotonously increasing function of temperature and no discontinuity is observed. These data allow us to fix a triple point between the solidus/liquidus and the congruent melting line of AMH-VII at $324 \mathrm{~K}$ and $3.59 \mathrm{GPa}$ as illustrated in Fig. 5. In the explored $P-T$ range, the temperature of the congruent melting line appears, within uncertainties, to increase linearly with pressure with a slope of $74 \mathrm{~K} / \mathrm{GPa}$ up to $700 \mathrm{~K}$. 
TABLE I. Experimental melting points of AMH. $P_{m}$ is in GPa and $T_{m}$ is in K. Number in brackets correspond to the estimated uncertainties for $P_{m}$.

\begin{tabular}{|cc|cc|cc|}
\hline \hline$P_{m}$ & $T_{m}$ & $P_{m}$ & $T_{m}$ & $P_{m}$ & $T_{m}$ \\
\hline $3.59(24)$ & 324 & $5.64(12)$ & 476 & $7.12(17)$ & 578 \\
$3.96(17)$ & 353 & $6.02(17)$ & 496 & $7.42(28)$ & 603 \\
$4.45(15)$ & 376 & $6.41(14)$ & 528 & $7.69(28)$ & 629 \\
$4.80(15)$ & 413 & $6.54(14)$ & 546 & $8.10(32)$ & 650 \\
$5.30(15)$ & 460 & $6.56(19)$ & 552 & $8.34(37)$ & 675 \\
$5.36(12)$ & 437 & $6.96(22)$ & 568 & & \\
\hline \hline
\end{tabular}

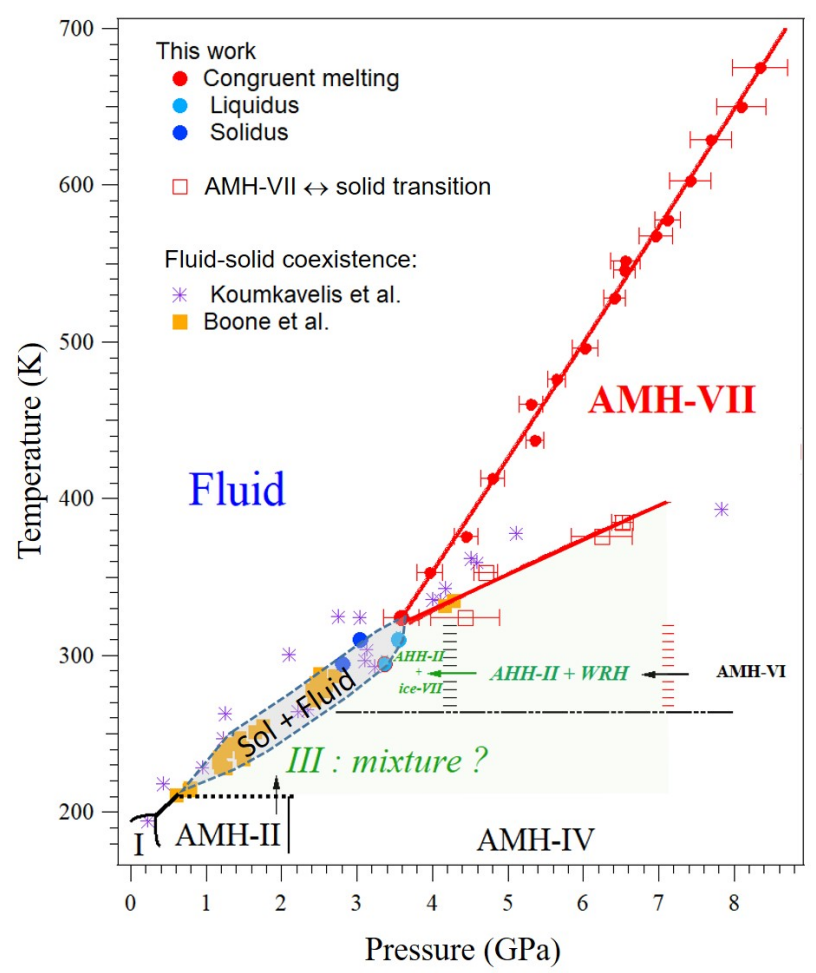

FIG. 4. (Color online) Phase diagram of AMH based on present work and literature. The purple and orange symbols represent the $(\mathrm{P}, \mathrm{T})$ points where a fluid/solid coexistence were reported respectively by Koumkavelis and $\mathrm{Nicol}^{29}$ and Boone and $\mathrm{Nicol}^{30}$. The fluid-AMHI, fluid-AMH-II and AMH-I/AMH-II transitions lines, drawn with solid black lines, are taken from Ref. [38]. The progressive dehydratation of AMH-VI at $295 \mathrm{~K}$ is observed here on decompression into $\mathrm{AHH}-\mathrm{II}+\mathrm{a}$ water-rich hydrate $(\mathrm{WRH})$ for $4.5<P<7 \mathrm{GPa}$ and into AHH-II + ice VII below 4.5 GPa. The solidus and liquidus $(\mathrm{P}, \mathrm{T})$ points below $324 \mathrm{~K}$ are indicated with respectively light and dark blue symbols. The liquidus and solidus lines, drawn with dotted grey lines, are based on present and Bonne and Nicol's data. Between these two curves, the solid-fluid coexistence is depicted by the grey zone. The light-green-colored domain indicate the $\mathrm{P}-\mathrm{T}$ range where solid solutions are observed. Above $324 \mathrm{~K}$, the red solid symbols and red line indicate the $(\mathrm{P}, \mathrm{T})$ points associated to the congruent melting and their fit by a Simon-Glatzel equation, respectively. The opened red square symbols represent the $(\mathrm{P}, \mathrm{T})$ solid/solid (AMH-VII/AHHII+WRH) transition line reported in the present work.

\section{Stability domain of AMH-VII}

As seen above, the presence of AMH-VII is only detected above the temperature of the triple point where the solidus, liquidus and melting line meet ( $324 \mathrm{~K}, 3.59 \mathrm{GPa})$. The Raman spectra of AMH-VII is clearly different from any of the solid phases observed at $295 \mathrm{~K}$ (AHH-II + ice VII or AHHII+WRH or AMH-VI). We have thus determined the transition from AMH-VII to other solid phases using Raman spectroscopy, along either isothermal (de)compression or quasiisobaric heating/cooling paths. The measured $(\mathrm{P}, \mathrm{T})$ points of the solid-solid transition line are reported in Fig. 5. As can be seen, the stability field of AMH-VII is delimited by the melting line at low pressure and the solid-solid transition line. We note that, in this $P-T$ range, the solid-solid transition line coincides with the dehydration of AMH into AHH-II + WRH. The transition/dehydration temperature appears to increase linearly with pressure with a slope of $21 \mathrm{~K} / \mathrm{GPa}$ up to 7 GPa.

\section{DISCUSSION}

\section{A. Observation of a new water-rich hydrate coexisting with AHH-II}

As seen above, an unidentified hydrate was observed to coexist with $\mathrm{AHH}$-II from $4.5 \mathrm{GPa}$ to $7 \mathrm{GPa}$ at ambient temperature on decompression from the DIMA phase. Its coexistence with $\mathrm{AHH}$ implies that this hydrate is richer in water than AMH and we thus named it water-rich hydrate (WRH). This WRH was only, but systematically, observed on decompression of the bcc DIMA phase, as the compression of the equimolar liquid leads to a solid mixture of AHH-II and ice VII ${ }^{39}$, remaining stable in compression up to at least 30 $\mathrm{GPa}^{40}$. We have not found a reasonable solution to the structure of this phase yet, but present below the current state of our analysis.

First, the observed XRD reflections show that WRH is not a $b c c$ phase, which excludes ice VII "doped" with ammonia and the disordered alloys (DIMA or DMA) reported in the literature $^{22,41-43}$. As a matter of fact the strongest diffraction peak assigned to the WRH at $5.1 \mathrm{GPa}$, is a doublet at $2 \theta=8.962^{\circ}$ and $9.043^{\circ}$ (corresponding to $d=2.392 \AA$ and $2.371 \AA$ ), where a single (110) peak is expected in a $b c c$ structure. If we consider this doublet as due to a slight tetragonal distortion of a $b c c$ cell with $Z=2$ as in ice VII or DIMA, we may estimate an average cubic lattice parameter $a=2.38 \pm 0.015 \AA$ at $5.1 \mathrm{GPa}, 300 \mathrm{~K}$, corresponding to a volume $V=19.05 \pm 0.3 \AA^{3} /$ molecule. As seen in Fig. 5, this volume is larger than that of ice VII and close to the one of ammonia dihydrate (ADH).

Second, WRH is not a known or predicted structure of ADH. At 5.1 GPa and ambient temperature, the stable state of the $2 \mathrm{H}_{2} \mathrm{O}: 1 \mathrm{NH}_{3}$ mixture is actually not an ADH phase, but AHH-II + ice VII ${ }^{42}$. We however compared the Bragg peaks of WRH to those of ADH-IV ${ }^{42}$, stable at low T, and found no correspondence. We also tested several predicted structures 
for ADH in the literature. The $P 2_{1} / n$ and $P 2_{1} 2_{1} 2_{1}$ structures from Ref. ${ }^{44}$ and the ADH-I* and ADH-II* structures reported in Ref. ${ }^{45}$ did not provide a reasonable fit. The closest match with our data was obtained with the $I 4_{1} c d$ structure predicted stable above $11.8 \mathrm{GPa}$ in Ref. ${ }^{44}$, and above $5 \mathrm{GPa}$ in Ref. ${ }^{45}$. As in this partially ionic structure, the molecular/ionic centers are arranged on a quasi-bcc lattice, we could obtain a correct Le Bail fit (see Fig.1(a) and (b) of the $\mathrm{SM}^{37}$ ) and the refined volume $\left(19.17 \AA^{3} /\right.$ molecule) is close to that expected for ADH $\left(19.60 \AA^{3} /\right.$ molecule). Nonetheless the two relatively strong (211) and (213) peaks of $I 4_{1} c d$ at low angles are not observed (see Fig.1(b) of the $\mathrm{SM}^{37}$ ). Furthermore, $I_{1} c d$ is a partially ionic structure (it is composed of $\mathrm{H}_{2} \mathrm{O}, \mathrm{NH}_{4}^{+}$and $\mathrm{OH}^{-}$) and a IR active $\mathrm{OH}^{-}$vibron is predicted ${ }^{45}$ with a frequency of $\sim 3710 \mathrm{~cm}^{-1}$. This vibron was observed in the DIMA phase of AMH above $7.4 \mathrm{GPa}^{22}$ but it disappears below this pressure. We therefore conclude that WRH is not $I 4_{1} c d$. It could correspond to a molecular version of this structure (if stable), or to another distorted $b c c$ structure yet to be found. Further work is thus needed to determine the structure of this phase.

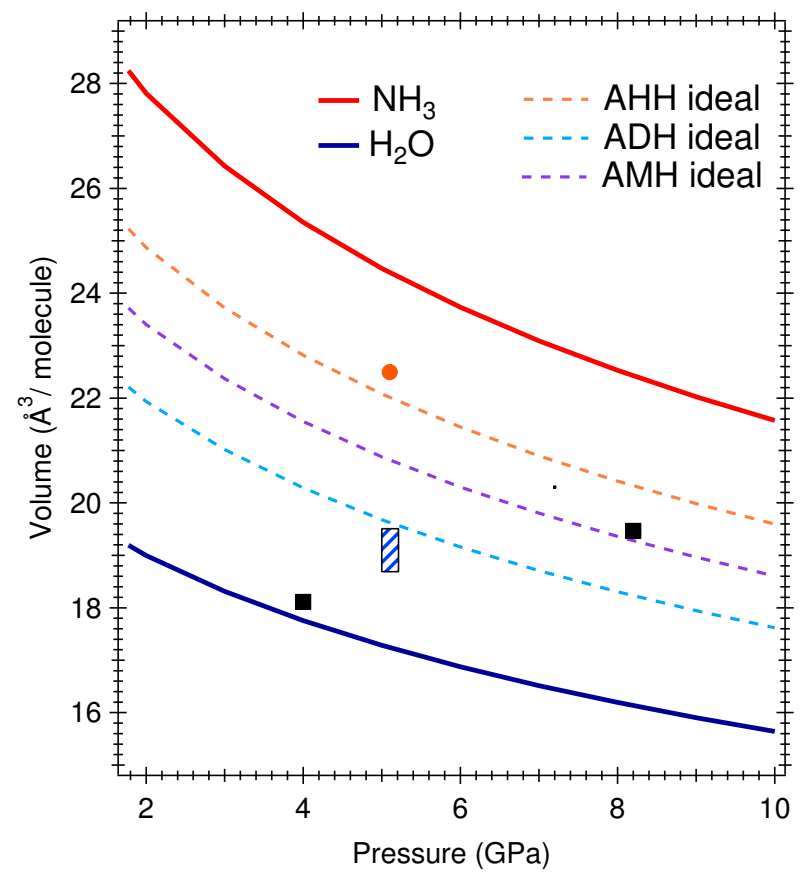

FIG. 5. (Color online) Comparison of the EOS of pure water, ammonia and ideal ammonia hydrates. The EOS of ammonia hydrates have been calculated by considering additivity of the partial volume of water $^{46}$ and ammonia ${ }^{47} \mathrm{~V}\left(\mathrm{xH}_{2} \mathrm{O}, y \mathrm{NH}_{3}\right)(\mathrm{P})=x /(x+y) \mathrm{V}_{\mathrm{H}_{2} \mathrm{O}}(\mathrm{P})+$ $\left.y /(x+y) V_{\mathrm{NH}_{3}}(P)\right)$. The symbols show the volume obtained in the present work at 8.2, 5.1 and $4 \mathrm{GPa}$.

\section{B. Congruent vs non-congruent melting}

The melting data of AMH obtained in this work is compared with previous experimental reports ${ }^{29,30}$ from $220 \mathrm{~K}$ to $400 \mathrm{~K}$ in Figure 5. In both studies, the authors reported the
$(\mathrm{P}, \mathrm{T})$ points where they visually observed a fluid/solid coexistence. Koumvakalis and $\mathrm{Nicol}^{29}$ only found a single solid phase in the range 120-450 K, 0-13.5 GPa, and thus concluded that melting should be congruent. We know by now that there is several solid phases in this P-T range. Furthermore, the pressure dispersion of their coexistence data points between about 260 and $320 \mathrm{~K}$ would actually suggest a non-congruent melting in this temperature interval. Boone and $\mathrm{Nicol}^{30}$ also reported that between 1 and $3 \mathrm{GPa}(220-280 \mathrm{~K})$, the coexistence was observed over a finite $(\sim 20 \mathrm{~K})$ temperature interval, indicative of a non-congruent melting. Combining these two previous works with the present one, we may thus conclude that melting is not congruent between $220 \mathrm{~K}$ and $324 \mathrm{~K}$. We plot the liquidus and solidus lines in Fig. 5 based on the present data and those of Bonne and Nicol which seem more consistent with each other.

Above $320 \mathrm{~K}$, the absence of dispersion in the coexistence data of previous and present work points to a congruent melting. Nonetheless, the melting points of Refs. [29 and 30] strongly deviate from ours. As a matter of fact they better agree with the transition points between AMH-VII and other solid phases found here. The discrepancy could thus be explained if the authors of references [29] and [30] confused melting with the transition to the high temperature phase AMH-VII. Indeed AMH-VII is visually very similar to the liquid phase: the two phases are transparent and their refractive indices are close. Moreover, their Raman spectra are also very close. Koumvakalis and Nicol actually write that "melting was detected visually as a sudden clearing of the polycrystalline samples with obvious reliefs and cracks". As reported above, we made exactly the same observation at the transition from the mixed phases to AMH-VII occuring before melting.

\section{Implication for AMH-III}

As seen in Figure 5, the change in melting behavior from congruent to non-congruent at $210 \mathrm{~K}$ coincide with the transition from AMH-II to AMH-III. AMH-II, whose structure was determined in Ref. 24 is a proper $\mathrm{AMH}$ phase, like $\mathrm{AMH}-$ $\mathrm{I}^{48}$. Consistently, its melting line is congurent, as measured by Ref. 38. AMH-III was discovered by heating AMH-II at $1.1 \mathrm{GPa}$ above $210 \mathrm{~K}$ in neutron diffraction experiments ${ }^{23}$. Its structure could not be disclosed from the measured diffraction pattern. The non-congruent melting strongly indicates that AMH-III is actually a mixed solid. Note that the latter was already conjectured by Loveday et $\mathrm{al}^{23}$, based on the complexity of the neutron pattern of AMH-III, and the irreversible character of the transitions between phases III, II and IV. We may thus safely conclude, that from $210 \mathrm{~K}$ to $324 \mathrm{~K}$, the equimolar fluid dehydrates upon freezing. Since the ambient T solid is composed of $\mathrm{AHH}$ and ice, and there is no, within uncertainties, detectable change of slope of the liquidus and solidus lines, we may suppose that the mixed solid referred as AMHIII is also a mixture of $\mathrm{AHH}$ and ice, or $\mathrm{AHH}$ and a water-rich hydrate. The transition observed by Loveday et $\mathrm{al}^{23}$ at 2.7 $\mathrm{GPa}, 270 \mathrm{~K}$ from III to $\mathrm{Vb}$ (now known to be AHH-II + ice VII), could originate from the VI-VII transition in ice, or the 
dehydration of the water-rich hydrate. This question should be addressed in future experiments.

\section{Comparison with water and ammonia}

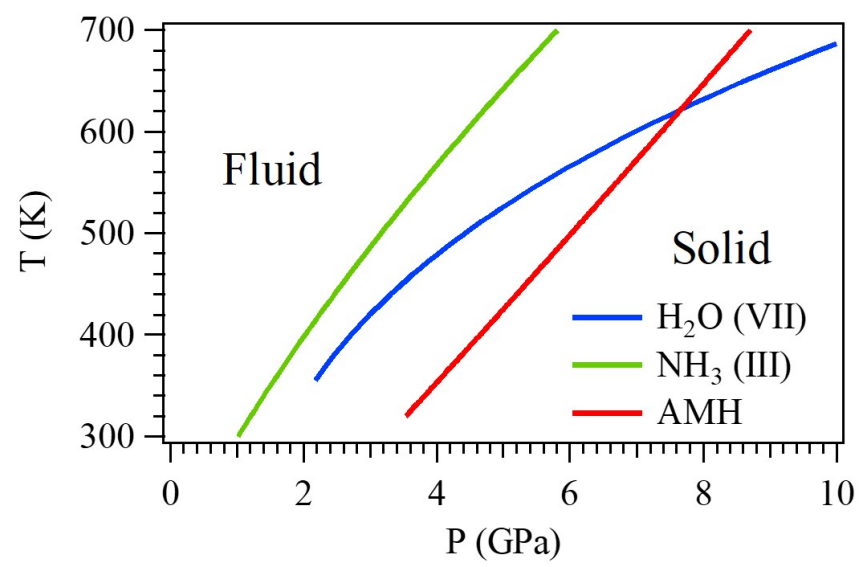

FIG. 6. (Color online) Comparison between the melting lines of the isoelectronic solids $\mathrm{H}_{2} \mathrm{O}^{33}, \mathrm{NH}_{3}{ }^{49}$ and $\mathrm{AMH}$.

The high temperature congruent melting line of $\mathrm{AMH}$ is compared with those of pure water ${ }^{33}$ and pure ammonia ${ }^{49}$ in Figure 6. Within uncertainties, the melting temperature of AMH grows linearly with pressure in the probed range, and thus presents a smaller concavity than water and ammonia. It can be seen, however, that the slope $d T_{m} / d P_{m}$ of the melting line of $\mathrm{AMH}$ is closer to that of $\mathrm{NH}_{3}$ than to the one of $\mathrm{H}_{2} \mathrm{O}$ at high P-T. This may indicate that the intermolecular interactions in the high temperature solid AMH-VII are closer to those in the pure $\mathrm{NH}_{3}$ solid than in the pure $\mathrm{H}_{2} \mathrm{O}$ solid.

\section{v. CONCLUSIONS}

In conclusion, the present work revises and extends the experimental phase diagram and melting properties of $\mathrm{AMH}$ up to $10 \mathrm{GPa}$, covering the previously unexplored hightemperature domain. The melting behavior has been disclosed up to $8.3 \mathrm{GPa}$ and $675 \mathrm{~K}$. Below $324 \mathrm{~K}$, melting is noncongruent as the stable solid before melting is a mixture of AHH-II and ice VII. We also observed that on decompression, AMH-VI first dehydrates around $7 \mathrm{GPa}$ into $\mathrm{AHH}-\mathrm{II}$ and an unknown, presumably water-rich hydrate, which itself decomposes into AHH-II and ice VII around $4 \mathrm{GPa}$. By combining previous and present data, we have proposed solidus and liquidus lines extending from $210 \mathrm{~K}$ to $324 \mathrm{~K}$, and implied that AMH-III is also a mixed phase, likely composed of $\mathrm{AHH}$ and ice, or $\mathrm{AHH}$ and a water-rich hydrate. Above $324 \mathrm{~K}$, melting is congruent, therefore the solid in equilibrium with the fluid has the same stoichiometry as the latter, i.e, it is a proper AMH phase, which we called AMH-VII. The Raman spectra of AMH-VII is distinct from all other known phases of AMH.
Its stability domain has been determined, and is delimited by the melting line at high temperature, and the dehydration line at low temperature. Comparison of the melting line of $\mathrm{AMH}-$ VII with those of $\mathrm{NH}_{3}$ and $\mathrm{H}_{2} \mathrm{O}$ suggests that the AMH-VII solid is more alike the plastic solid phase III of $\mathrm{NH}_{3}$. It is worth noting that plastic solid forms of ammonia hydrates have been recently observed in simulations close to the melting line ${ }^{28}$. Future work should thus now focuss on deciphering the structural and dynamical properties of AMH-VII, and extend the determination of the phase diagram to higher P-T conditions.

\section{ACKNOWLEDGMENTS}

We thank G. Garbarino and V. Svitlyk for assistance in the XRD experiments at ESRF. We acknowledge IMPMC for the use of the spectroscopy platform and ESRF for the provision of beam time allocated to proposals HC-2483 and HC3685. This work was financially supported by the Agence Nationale de la Recherche under grants ANR-15-CE30-000801 (SUPER-ICES), the Chinese Scholarship Council through the allocation of a scholarship to Haiwa ZHANG, and French state funds managed by the ANR within the Investissements d'Avenir programme under reference ANR-11-IDEX-000402, and more specifically within the framework of the Cluster of Excellence MATISSE led by Sorbonne Université.

\section{DATA AVAILABILITY STATEMENTS}

The data that support the findings of this study are available from the corresponding author upon reasonable request.

\section{SUPPLEMENTARY MATERIALS}

See the supplementary materials ${ }^{37}$ for additional figures of AMH XRD pattern and Raman spectra .

${ }^{1}$ W. B. Hubbard and J. J. M. Farlane, "Structure and evolution of Uranus and Neptune," J. Geophys. Res. 85, 225-234 (1980).

${ }^{2}$ W. B. Hubbard, "Interiors of the giant planets," Science 214, 145-149 (1981).

${ }^{3}$ T. Guillot, "Interiors of giant planets inside and outside the solar system," Science 286, 72-77 (1999).

${ }^{4}$ D. D. Sasselov, "Extrasolar planets," Nature 451, 29-31 (2008).

${ }^{5}$ R. Redmer, T. Mattsson, N. Nettelmann, and M. French, "The phase diagram of water and the magnetic fields of Uranus and Neptune," Icarus 211, 798-803 (2011).

${ }^{6}$ R. H. Brown and D. Cruikshank, "Determination of the composition and state of icy surfaces in the outer solar system." Annu. Rev. Earth Pl. Sc. 25, 243-277 (1997).

${ }^{7}$ C. Cavazzoni, G. L. Chiarotti, S. Scandolo, E. Tosatti, M. Bernasconi, and M. Parrinello, "Superionic and metallic states of water and ammonia at giant planet conditions," Science 283, 44-46 (1999).

${ }^{8}$ A. F. Goncharov, N. Goldman, L. E. Fried, J. C. Crowhurst, I.-F. Kuo, C. J. Mundy, and J. M. Zaug, "Dynamic ionization of water under extreme conditions," Phys. Rev. Lett. 94, 125508 (2005).

${ }^{9}$ J. A. Hernandez and R. Caracas, "Proton dynamics and the phase diagram of dense water ice," J. Chem. Phys. 148, 214501 (2018). 
${ }^{10}$ E. Sugimura, T. Komabayashi, K. Ohta, K. Hirose, Y. Ohishi, and L. S. Dubrovinsky, "Experimental evidence of superionic conduction in $\mathrm{H}_{2} \mathrm{O}$ ice," J. Chem. Phys 137, 194505 (2012).

${ }^{11}$ M. Millot, F. Coppari, J. R. Rygg, A. C. Barrios, S. Hamel, D. C. Swift, and J. H. Eggert, "Nanosecond x-ray diffraction of shock-compressed superionic water ice," Nature 569, 251 (2019).

${ }^{12}$ S. Ninet, F. Datchi, and A. Saitta, "Proton disorder and superionicity in hot dense ammonia ice," Phys. Rev. Lett. 108, 165702 (2012).

${ }^{13}$ C. Pickard and R. Needs, "Highly compressed ammonia forms an ionic crystal," Nat. Mater. 7, 775 (2008).

${ }^{14}$ S. Ninet, F. Datchi, P. Dumas, M. Mezouar, G. Garbariono, A. Mafety, C. Pickard, R. Needs, and A. Saitta, "Experimental and theoretical evidence for an ionic crystal of ammonia at high pressure," Phys. Rev. B 89, 174103 (2014).

${ }^{15}$ T. Palasyuk, I. Troyan, M. Eremets, V. Drozd, S. Medvedev, P. ZaleskiEjgierd, E. Magos-Palasyuk, H. Wang, S. A. Bonev, D. Dudenko, and P. Naumov, "Ammonia as a case study for the spontaneous ionization of a simple hydrogen-bonded compound," Nature Communications 5, 3460 (2014).

${ }^{16}$ W. Holzapfel, "On the symmetry of the hydrogen bonds in ice VII," J. Chem. Phys. 56, 712-715 (1972).

${ }^{17}$ K. Aoki, H. Yamawaki, M. Sakashita, and H. Fujihisa, "Infrared absorption study of the hydrogen-bond symmetrization in ice to $110 \mathrm{GPa}$," Phys. Rev. B 54, 15673 (1996).

${ }^{18}$ E. Wolanin, P. Pruzan, J. C. Chervin, B.Canny, M. Gauthier, D. Haüsermann, and M. Hanfland, "Equation of state of ice VII up to $107 \mathrm{GPa}$," Phys. Rev. B 56, 5781 (1997).

${ }^{19}$ M. Benoit, D. Marx, and M. Parrinello, "Tunnelling and zero-point motion in high-pressure ice," Nature 392, 258 (1998).

${ }^{20}$ A. F. Goncharov, V. V. Struzhkin, H. K. Mao, and R. J. Hemley, "Raman spectroscopy of dense $\mathrm{H}_{2} \mathrm{O}$ and the transition to symmetric hydrogen bonds," Phys. Rev. Lett. 83, 1998 (1999).

${ }^{21}$ J. S. Loveday and R. J. Nelmes, "Ammonia monohydrate VI: A hydrogenbonded molecular alloy," Phys. Rev. Lett. 83, 4329 (1999).

${ }^{22}$ C. Liu, A. Mafety, J. A. Queyroux, C. W. Wilson, H. Zhang, K. Béneut, G. L. Marchand, B. Baptiste, P. Dumas, G. Garbarino, F. Finocchi, J. S. Loveday, F. Pietrucci, A. M. Saitta, F. Datchi, and S. Ninet, "Topologically frustrated ionisation in a water-ammonia ice mixture," Nat. Commun. 8, 1065 (2017).

${ }^{23}$ J. S. Loveday and R. J. Nelmes, "The ammonia hydrates : model mixed hydrogen-bonded systems," High Pressure Research 24, 45 (2004).

${ }^{24}$ A. D. Fortes, E. Suard, M. H. Lemée-Cailleau, C. J. Pickard, and R. J. Needs, "Crystal structure of ammonia monohydrate phase II," J. Am. Chem. Soc. 131, 13508-13515 (2009).

${ }^{25} \mathrm{C}$. W. Wilson, C. L. Bull, G. Stinton, and J. S. Loveday, "Pressure-induced dehydration and the structure of ammonia hemihydrate-II," J. Chem. Phys. 136, 094506 (2012)

${ }^{26}$ G. I. G. Griffiths, A. J. Misquitta, A. D. Fortes, C. J. Pickard, and R. J. Needs, "High pressure ionic and molecular crystals of ammonia monohydrate within density functional theory," J. Chem. Phys. 137, 064506 (2012).

${ }^{27}$ M. Bethkenhagen, D. Cebulla, R. Redmer, and S. Hamel, "Superionic phases of the 1:1 water-ammonia mixture," J. Phys. Chem. A 119, 10582 (2015).

${ }^{28}$ V. N. Robinson and A. Hermann, "Plastic and superionic phases in ammonia water mixtures at high pressures and temperatures," J. Phys. Condens. Matter 32, 184004 (2020).

${ }^{29} \mathrm{~A}$. Koumvakalis and M. Nicol, "Raman spectroscopy of ammonia monohydrate to 13.5 GPa," High Pressure Chemistry, Biochemistry and Materials
Science, 265 (1993).

${ }^{30} \mathrm{~S}$. Boone and M. E. Nicol, "Ammonia-water mixtures at high pressures : melting curves of ammonia dihydrate and ammonia monhydrate and a revised high pressure phase diagram for the water rich region," Proceeding of lunar and planetary science 21, 606 (1991).

${ }^{31}$ P. I. Dorogokupets and A. R. Oganov, Phys. Rev. B 75, 024115 (2007).

${ }^{32}$ F. Datchi, R. LeToullec, and P. Loubeyre, "Improved calibration of the $\mathrm{SrB}_{4} \mathrm{O}_{7}: \mathrm{Sm}^{2}+$ optical pressure gauge: Advantages at very high pressure and high temperatures," J. Appl. Phys. 81, 3333 (1997).

${ }^{33}$ F. Datchi, P. Loubeyre, and R. LeToullec, "Extended and accurate determination of the melting curves of argon, helium, ice $\mathrm{H}_{2} \mathrm{O}$ and hydrogen $\mathrm{H}_{2}$," Phys. Rev. B 61, 6535-6546 (2000).

${ }^{34}$ A. Hammersley, S. O. Svensson, M. Hanfland, A. Fitch, and D. Hausermann, "Two dimensional detector software : From real detector to idealised image or two-theta scan.” High Press. Res. 14, 235 (1996).

${ }^{35} \mathrm{C}$. Prescher and V. B. Prakapenka, "Dioptas : a program for reduction of two-dimensional x-ray diffraction data and data exploration," High Press. Res. 35, 223 (2015).

${ }^{36} \mathrm{~J}$. Rodríguez-Carvajal, "Recent advances in magnetic structure determination by neutron powder diffraction," Physica B 192, 55-69 (1993).

${ }^{37}$ H. Zhang, F. Datchi, L. Andriambariarijaona, G. Zhang, J. A. Queyroux, K. Béneut, M. Mezouar, and S. Ninet, "Supplementary materials of "melting curve and phase diagram of ammonia monohydrate at high pressure and temperature"," J. Chem. Phys (2020).

${ }^{38}$ D. L. Hogenboom, J. S. Kargel, G. J. Consolmagno, T. C. Holden, L. Lee, and M. Buyyounouski, "The ammonia water system and the chemical differentiation of icy satellites," Icarus 128, 171 (1997).

${ }^{39}$ C. W. Wilson, C. L. Bull, G. W. Stinton, D. M. Amos, M.-E. Donnelly, and J. S. Loveday, "On the stability of the disordered molecular alloy phase of ammonia hemihydrate," J. Chem. Phys. 142, 094707 (2015).

${ }^{40} \mathrm{X}$. Li, W. Shi, X. Liu, and Z. Mao, "High-pressure phase stability and elasticity of ammonia hydrate," American Mineralogist 104, 1307 (2019).

${ }^{41}$ J. S. Loveday, R. J. Nelmes, W. G. Marshall, J. M. Besson, S. Klotz, and G. Hamel, "Structure of deuterated ammonia IV," Phys. Rev. Lett. 76, 74 (1996).

${ }^{42}$ A. D. Fortes, I. G. Wood, M. Alfredsson, L. Vojcadlo, K. S. Knight, W. G. Marshall, M. G. Tucker, and F. Fernandez-Alonso, "The high-pressure phase diagram of ammonia dihydrate," High Pressure Res. 27, 201-212 (2007).

${ }^{43}$ J. S. Loveday, R. J. Nelmes, C. L. Bull, H. E. Maynard-Casely, and M. Guthrie, "Observation of ammonia dihydrate in the AMH-VI structure at room temperature - possible implications for the outer solar system," High Pressure Research 29, 396-404 (2009).

${ }^{44} \mathrm{X}$. Jiang, X. Wu, Z. Zheng, Y. Huang, and J. Zhao, "Ionic and superionic phases in ammonia dihydrate $\mathrm{NH}_{3}-2 \mathrm{H}_{2} \mathrm{O}$ under high pressure," Phys. Rev. B 95, 144104 (2017).

${ }^{45}$ V. N. Robinson, M. Marques, Y. Wang, Y. Ma, and A. Hermann, "Novel phases in ammonia water mixtures under pressure," J. Chem. Phys. 149, 234501 (2018)

${ }^{46}$ S. Klotz, K. Komatsu, H. Kagi, K. Kunc, A. Sano-Furukawa, and S. M. andT. Hattori, "Bulk moduli and equations of state of ice VII and ice VIII," Phys. Rev. B 95, 174111 (2017).

${ }^{47}$ F. Datchi, S. Ninet, M. Gauthier, A. M. Saitta, B. Canny, and F. Decremps, "Solid ammonia at high pressure: A single-crystal x-ray diffraction study to $123 \mathrm{GPa}$," Phys. Rev. B 73, 174111 (2006).

${ }^{48}$ I. Olovsson and D. H. Templeton, "The crystal structure of ammonia monohydrate," Acta. Cryst. 12, 827 (1959).

${ }^{49}$ S. Ninet and F. Datchi, "High pressure-high temperature phase diagram of ammonia." J Chem Phys 128, 154508 (2008). 